Ann. Zootech., I96r, 10 (2), 69-87.

\author{
I.N.R.A. \\ BIBLIOTHEQUE UD :Eg \\ Domaine de Crovel. \\ 63039 \\ Clermont-Fo Cegex \\ 712102
}

\title{
ÉTUDE SUR LES VARIATIONS DES TENEURS EN MATIÈRES AZOTÉES ET EN ÉLÉMENTS MINÉRAUX DE LA FÉTUQUE DES PRÉS
}

\author{
L. GUEGUEN et G. FAUCONNLAU \\ Avec la collaboration technique de Madeleine Forignon et de Valérie François-Goujon \\ Service de Biochimie et de Nutrition, \\ Centre national de Recherches zootechniques, Jouy-en-Josas (Seine et Oise).
}

\section{SOMMAIRE}

Les variations des teneurs en matières azotées et en éléments minéraux ( $\mathrm{P}, \mathrm{Ca}, \mathrm{K}, \mathrm{Na}, \mathrm{Mg}$ ) d'une graminée fourragère, la Fétuque des prés, ont été étudiées après séparation des différentes parties de la plante : limbes, gaines, tiges, fleurs et débris. La Fétuque a été récoltée à trois stades de développement différents durant plusieurs cycles de végétation au cours de l'année 1959.

Cette étude complète des travaux effectués en r957 et I958 sur le Dactyle et la Fétuque et permet de confirmer quelques lois générales déjà énoncées.

Au cours du premier cycle, les variations de la composition chimique des plantes sont étroitement liées à l'évolution du rapport limbes/gaines + tiges et aux translocations provoquées par la floraison. Les variations de la composition des plantes du premier cycle semblent peu sensibles aux facteurs externes (climat, sol...).

Au cours des cycles suivants la Fétuque est constituée principalement de limbes et les variations de la composition chimique de la plante sont beaucoup plus sensibles aux facteurs climatiques.

La comparaison des résultats obtenus sur le Dactyle en 1958 (année très humide) et sur la Fétuque en 1959 (année très sèche) a permis de mettre en évidence une influence climatique prépondérante sur les teneurs en matières azotées et en phosphore des plantes (considérablement diminuées par la sécheresse) et une influence saisonnière prépondérante sur les teneurs en calcium et en magnésium (qui augmentent au cours de l'année).

Les plantes du premier cycle ont en général la plus faible valeur nutritive en matières azotées et en éléments minéraux.

Dans le cadre des travaux entrepris sur la composition chimique des espèces pures de plantes fourragères, nous avons étudié les variations des teneurs en matières azotées et en éléments minéraux de la Fétuque des prés (Festuca pratensis), en fonction du stade de développement et du cycle de végétation, après séparation des différents organes de la plante. Cette expérience complète une étude antérieure sur le premier cycle de végétation d'un dactyle et d'une fétuque en I957 (GUEGUEN, I959) et suit un travail identique effectué en I958 sur le Dactyle (GuEguen et Fauconneau, r960). 


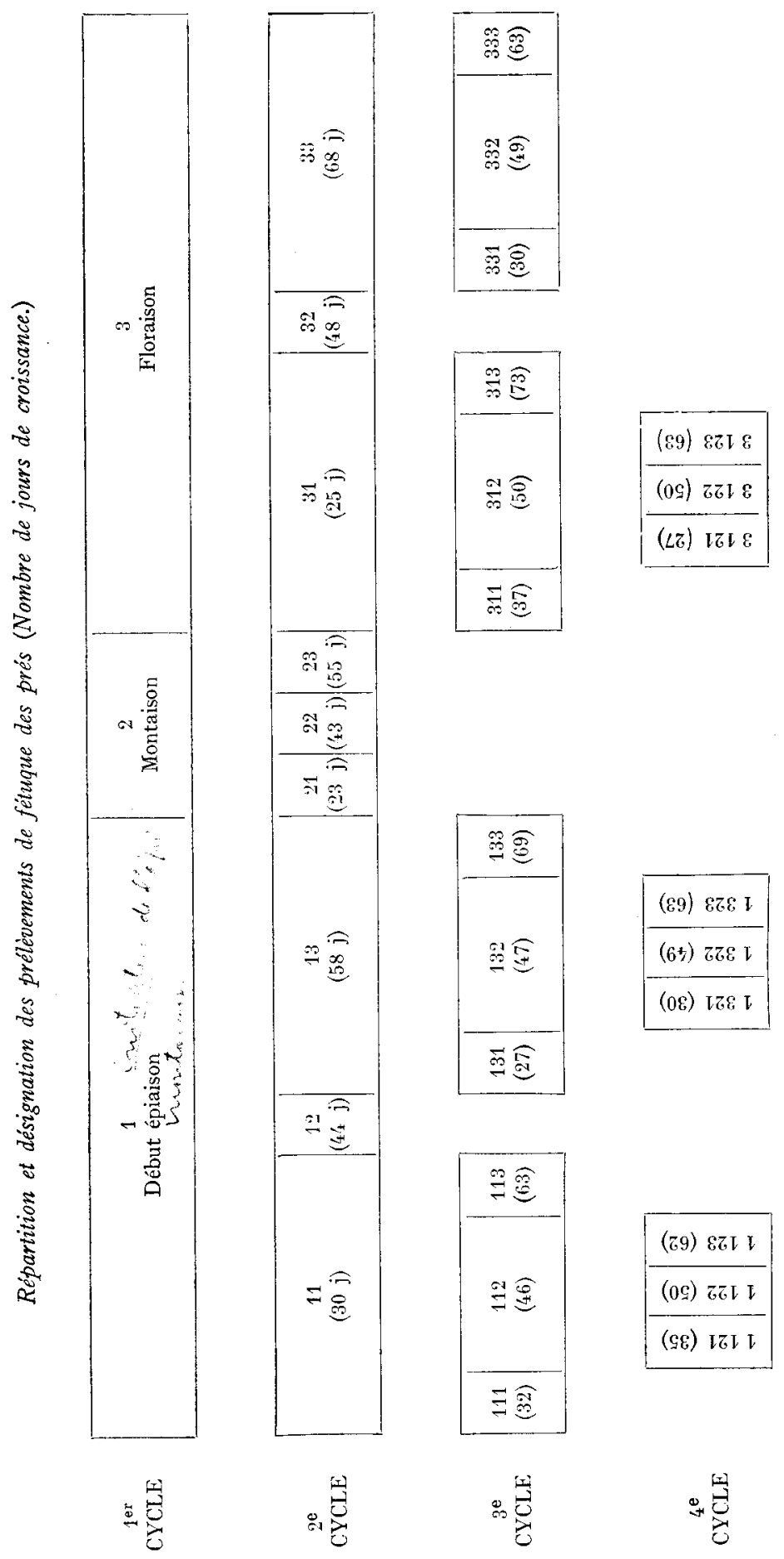


MATIÈRES AZOTÉES ET ÉLÉMENTS MINÉRAUX

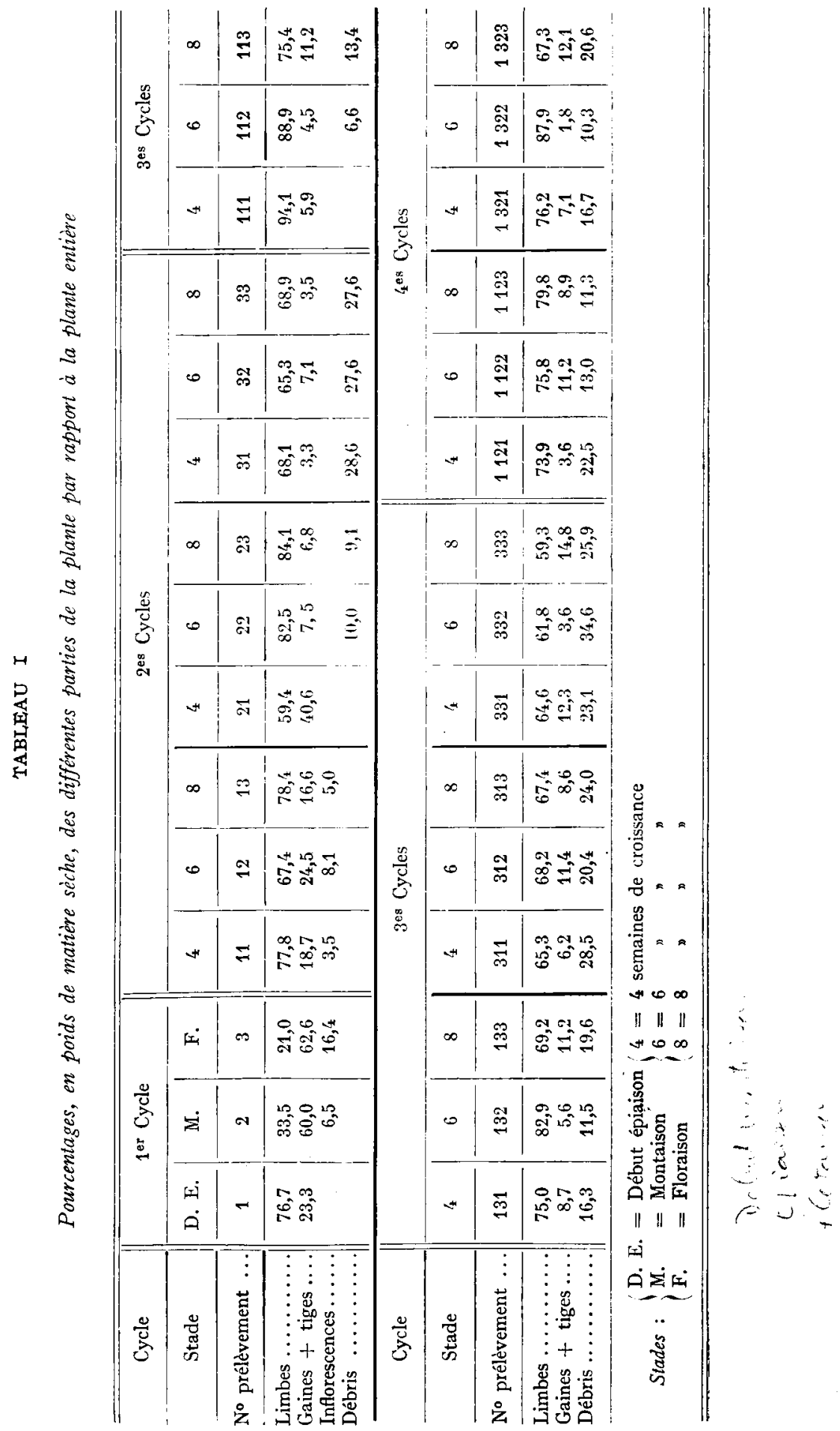


La comparaison de ces trois essais permet déjà de mettre en évidence l'influence de la saison et des conditions climatiques extrêmes sur les teneurs de certains constituants dans la plante.

\section{MATÉRIEL E'T MÉTHODES}

\section{Matériel expérimental:}

L'cspèce étudiée est une fétuque des prés semée au printemps $195^{8}$ sur une parcelledu C. N. R. Z. (sol argilo-calcaire), avant reçı une forte fumure azotée ( 80 unités par hectare et par coupe) et récoltée durant l'année 1959 à trois stades de développement durant plusieurs cycles de végétation. Les trois starles de récolte choisis corespondent pour le premier cycle au début de l'épiaison, à la montaison et a la floralson et, pour les cycles suivants à trois durées de croissance $: 4$ semaines, 6 semaines et 8 semaines.

Le protocole expérimc ntal, le mode de prélèvement des échantillons et la nomenclature adoptée pour désigner les coupes sontdétaillés dans l'étude effectuée sur le dactvle (Gi:EGLEN et FAUCONNEAU, I960). Nous avons ré:umé dans le tableau suivant la répartition et la désignation des prélèvements ${ }^{1}$ ).

\section{Traitement des échantillons et méthodes d'analyse:}

Après séparation des limbes, gaines, tiges, fleurs et débris, les proportions relatives de ces divers organes par rapport à la plante entière ont été déterminées en matière sèche après passage au four à circulation d'air à $60-70^{\circ} \mathrm{C}$. Les échantillons sont finement broyés et conservés en chambre froide à $0-2^{\circ} \mathrm{C}$. La teneur en matière sèche est déterminée avant les différents dosages par séjour à l'étuve à $102-105^{\circ} \mathrm{C}$ pendant environ I 5 heures. L.es matières azotées $(\mathrm{N} \times 6,25)$ ont été obtenues par la méthode de Kjeldahl et les éléments minéraux ont été dosés après calcination du produit à $53^{\circ} \mathrm{C}$ : le phosphore par la méthode colorimétrique au phosphovanadomolybdate d'ammonium et le calcium, le potassium, le sodium et le magnésium par spectrophotométrie de flamme (GitggueN et Rombauts, ig6i).

\section{RÉSULTATS}

\section{INTERPRÉTATIONS PHYSIOLOGIQUES}

Comme dans le cas du Dactyle, les plantes du premier cycle diffèrent profondément, du point de vue morphologique, des plantes des cycles suivants ; ces dernières sont constituées exclusivement de limbes et d'une très faible proportion de gaines. Ainsi, les variations de la composition chimique des plantes du premier cycle sont étroitement liées d'une part à l'évolution rapide du rapport limbes/gaines + tiges et d'autre part aux translocations provoquées par la floraison. En revanche, au cours des cycles suivants, les variations de la composition de la plante entière sont parallèles aux variations de la composition des limbes. Dans le cas de la Fétuque, étudiée en année sèche,

- le rapport limbes/gaines est très élevé (tableau I). Par contre, pour le dactyle étudié en année humide, la proportion de gaines n'était pas négligeable ( 15 à $20 \mathrm{p}$. IOo) et justifiait la séparation préalable des organes pour l'interprétation des résultats.

L'action de la sécheresse (en moyenne i mm de pluie par mois de mai à octobre) a considérablement diminué l'efficacité des engrais azotés et les rendements obtenus après le premier cycle ont été très inférieurs à ceux fournis par le dactyle en I958 (figure I).

(1) Exemple : la coupe numérotée $33^{2}$ a été récoltée au $2^{e}$ stade de développement 6 semaines de croissance), fait partie d'un $3^{\theta}$ cycle de végétation, repousse d'une coupe effectuée aul $3^{\mathrm{e}}$ stade d'un second cycle provenant lui-même du $\mathrm{I}$ er cycle interrompu au stade floraison. 


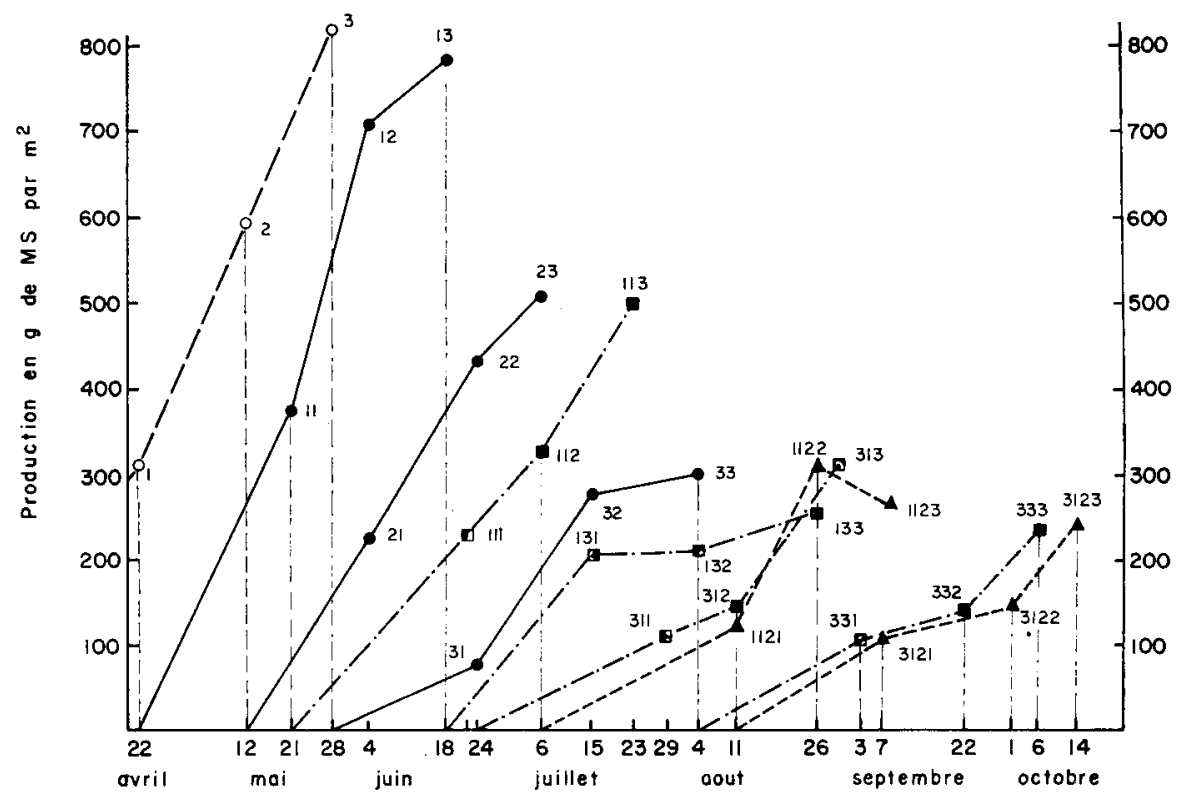

Fig. I. - Production de matière sèche par la fétuque des prés au cours des différents cycles.

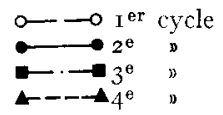

Légende commune à toutés les figures.

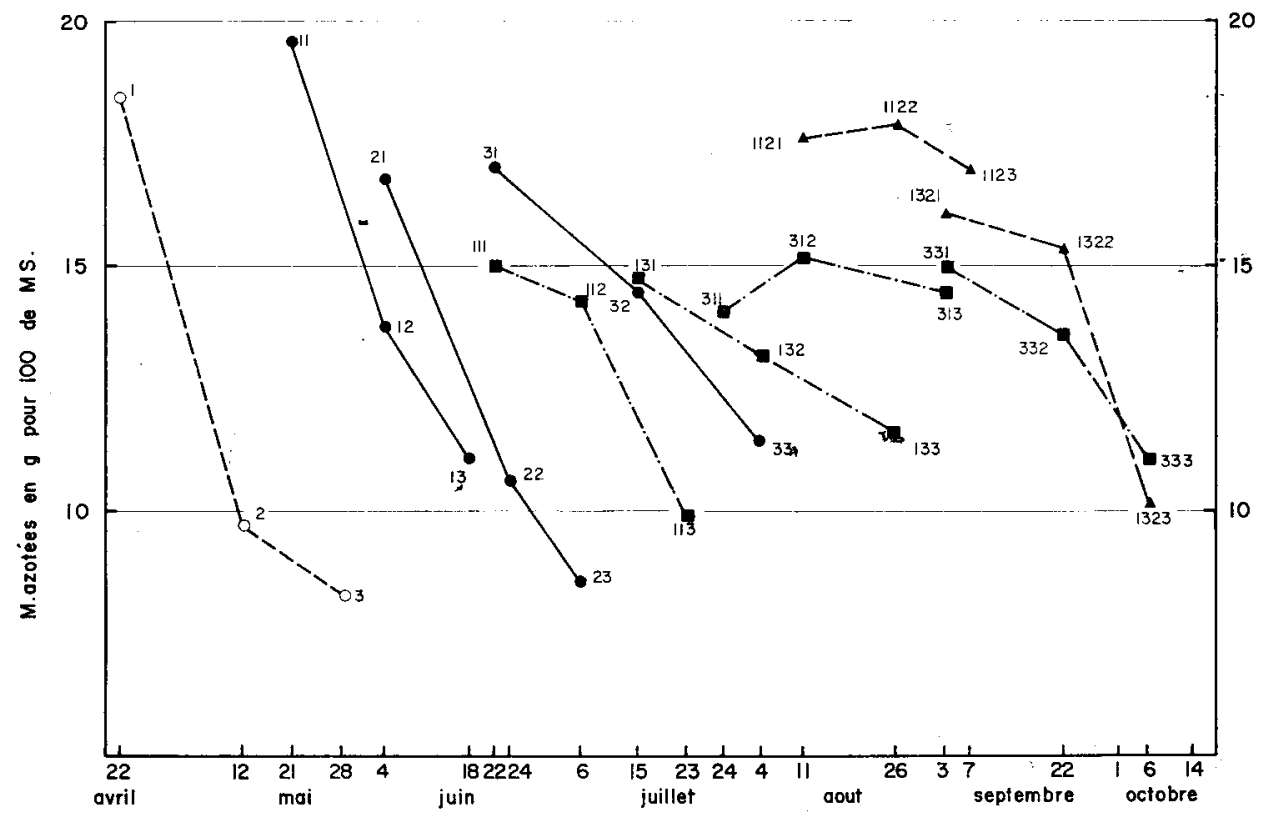

FIG. 2. - Evolution de la teneur en matières azolées $(N \times 6,25)$ de la fétuque des prés. 


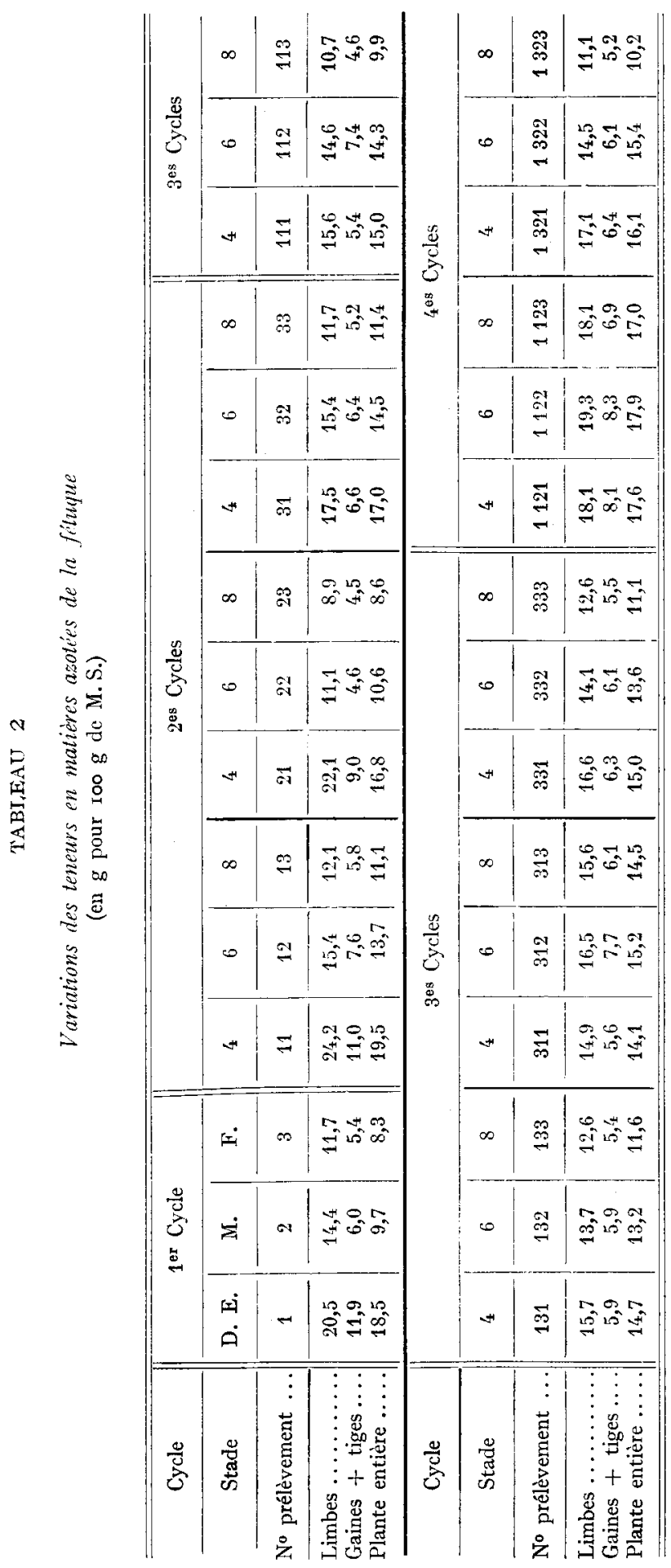


MATIÈRES AZOTÉES ET ÉLÉMENTS MINÉRAUX

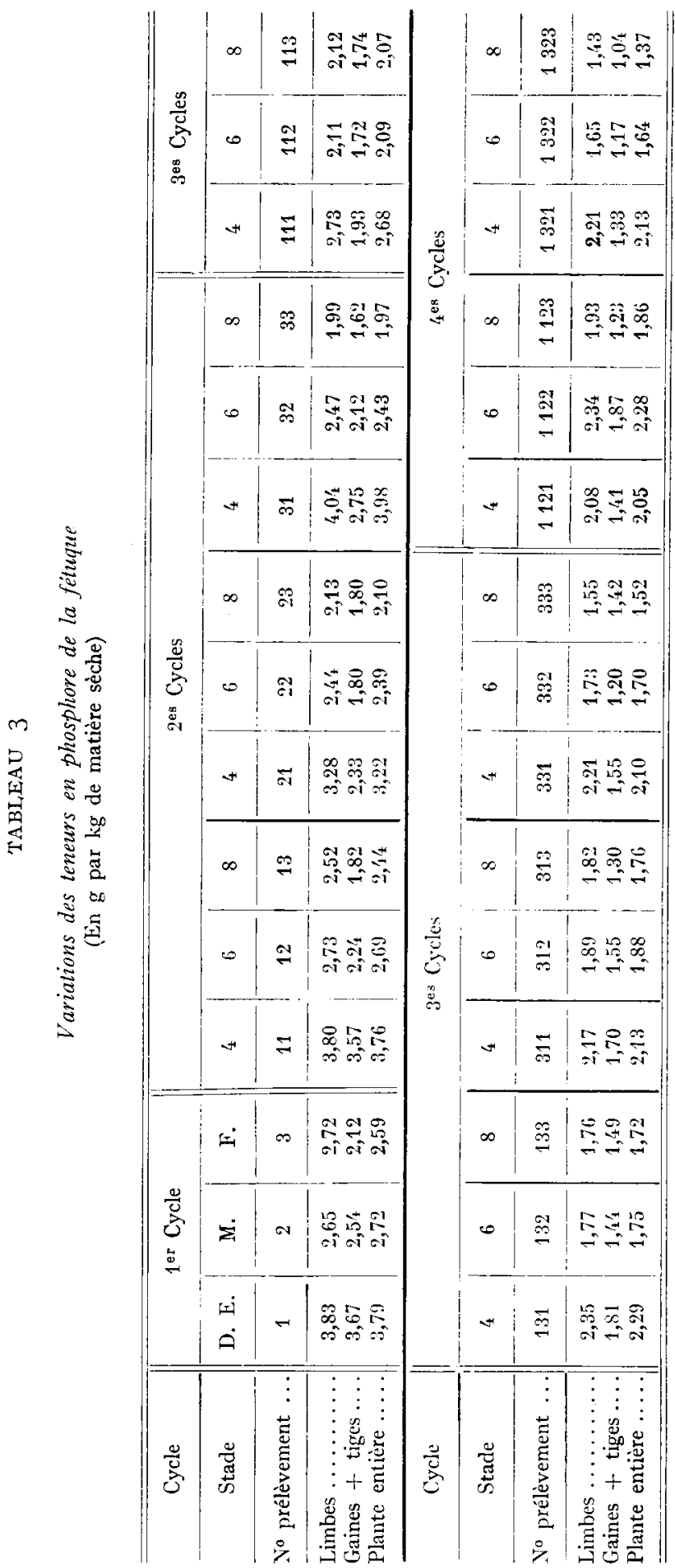


I. GUEGUEN, G. FAUCONNEAU

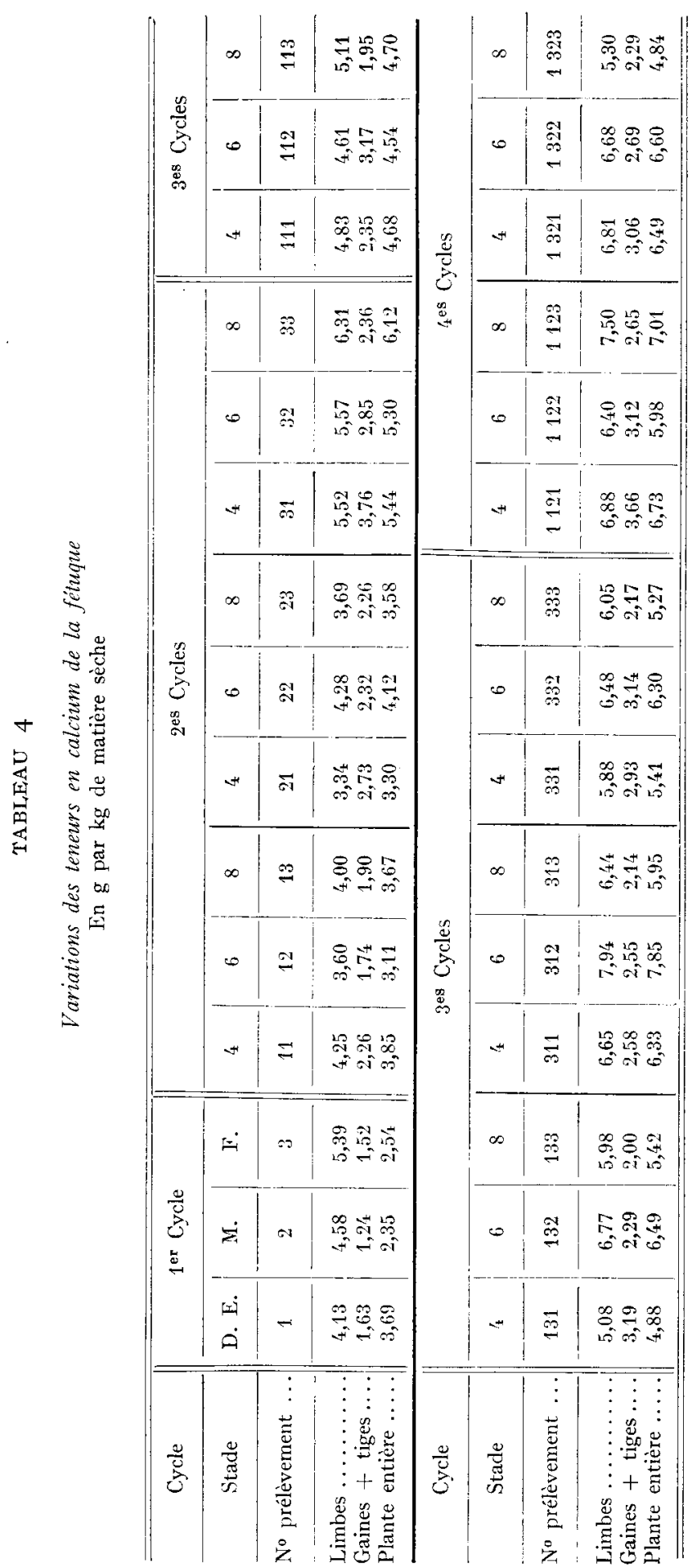




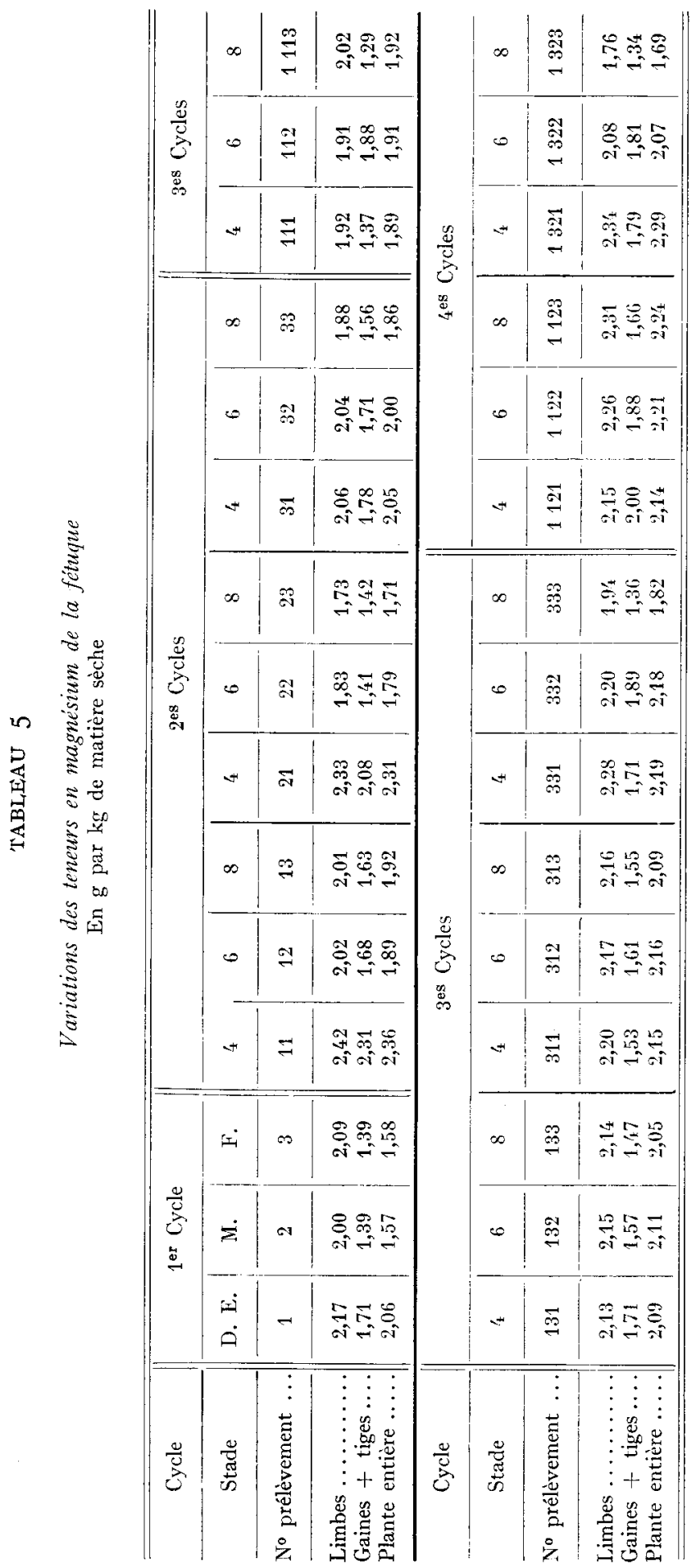


L. GUEGUEN, G. FAUCONNEAU

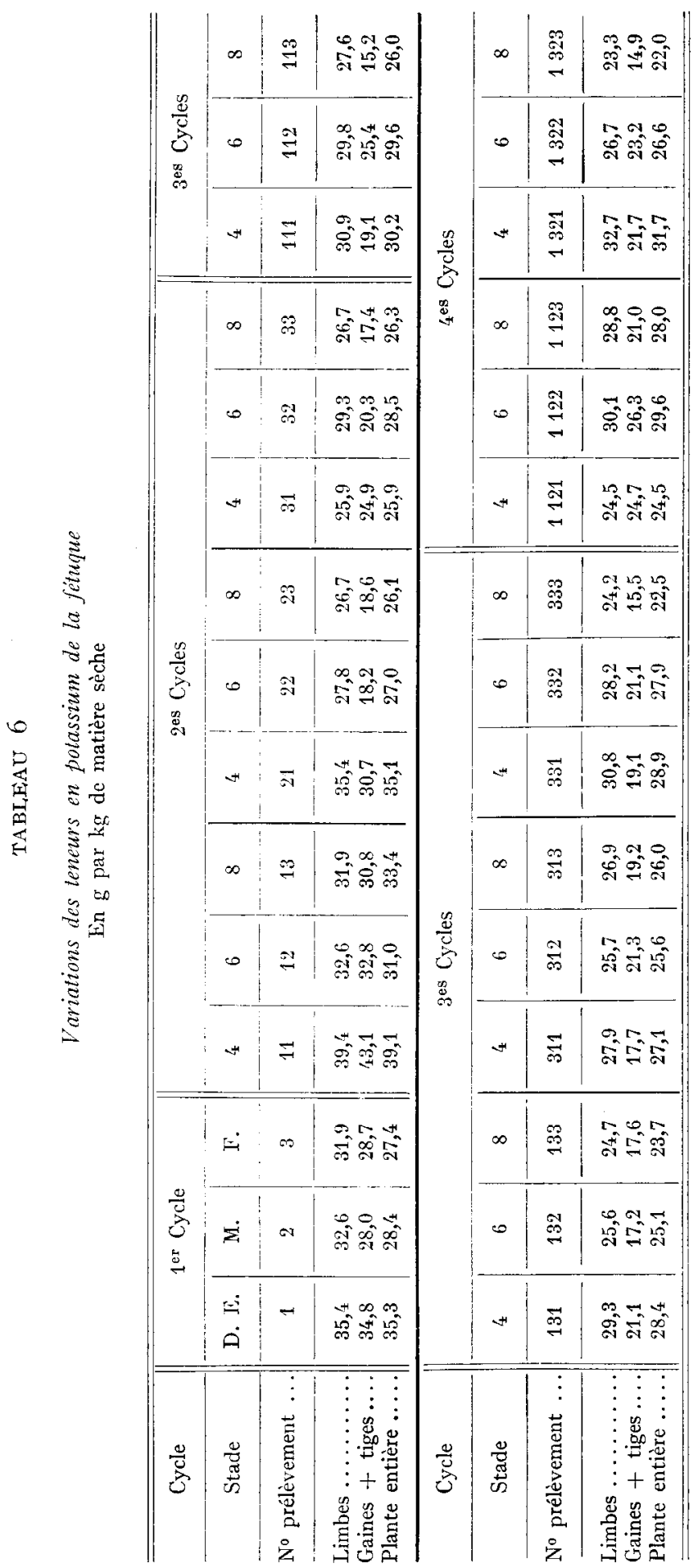




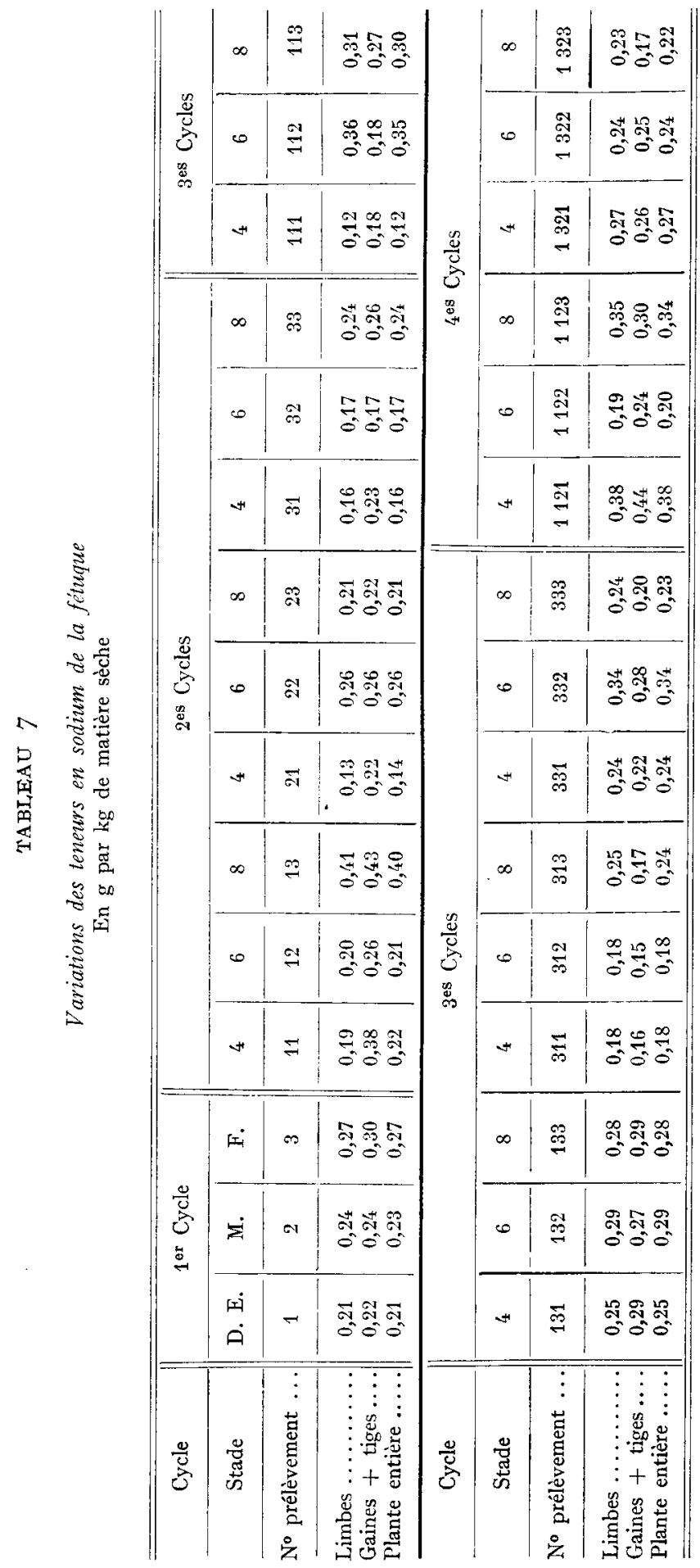




\section{I. - Les matières azotées $(\mathrm{N} \times 6,25)$}

Composition des divers organes et de la plante entière.

I es limbes, essentiellement constitués de parenchymes chlorophylliens (MITCHELI, et SOPER, I956), sont toujours beaucoup plus riches ( 2 à 2,5 fois) en matières azotées que les gaines et les tiges dans tous les cycles (tableau 2); les inflorescences sont assez riches (20 à I3 p. Ioo de la matière sèche).

Les plantes des $2^{e}, 3^{\mathrm{e}}$ et $4^{\mathrm{e}}$ cycles sont toujours plus riches en matières azotées que celles du $\mathrm{I}^{\mathrm{er}}$ cycle : en moyenne les fétuques âgées de 6 semaines contiennent I3 à I4 p. Ioo de matières azotées au lieu de 9,5 p. Ioo ( ${ }^{\text {er }}$ cycle).

\section{Influence du stade de développement.}

'Tous les organes s'appauvrissent régulièrement avec l'âge quel que soit le cycle: les matières azotées des limbes diminuent de 20 à Io p. Ioo (de M. S.) en quatre semaines. Au cours du premier cycle, les teneurs en matières azotées de la plante entière diminuent d'abord rapidement (par suite de la diminution importante de la proportion des limbes), puis plus lentement (par suite de l'importance grandissante des inflorescences). Au cours des cycles suivants les teneurs en matières azotées de la plante entière diminuent régulièrement comme dans les limbes (90 p. Ioo à $95 \mathrm{p}$. roo de la plante entière).

\section{2. - Le Phosphore}

Composition des divers organes et de la plante entière.

Nous retrouvons des règles générales énoncées antérieurement : les teneurs en $\mathrm{P}$ sont toujours plus élevées dans les limbes que dans les gaines et les tiges; les inflorescences sont particulièrement riches en $P$.

A stade botanique équivalent, limbes et gaines s'appauvrissent considérablement en $\mathrm{P}$ au cours de l'année (tableau 3). Ceci ne semble pas dû à une évolution physiologique normale mais est vraisemblablement la conséquence des conditions extrêmes de sécheresse de l'année 1959. Ainsi, contrairement à ce que nous avions observé pour le dactyle (année I958, très humide), les teneurs en $\mathrm{P}$ de la plante entière diminuent régulièrement au cours de l'année et sont particulièrement faibles en automne (figure 4).

\section{Infuence du stade de développement.}

De façon très générale les teneurs en $\mathrm{P}$ diminuent dans tous les organes avec le stade de développement. Si cette diminution est normale au cours du cycle reproductif de la plante, elle peut seulement s'expliquer, à partir du $2^{\mathrm{e}}$ cycle, par la forte sécheresse qui rend de plus en plus difficile la mobilisation du phosphore du sol par la plante âgée.

Au cours du premier cycle, la proportion croissante des inflorescences, très riches en $\mathrm{P}$, contribue à freiner la chute brutale de la teneur en $\mathrm{P}$ de la plante entière (tableau 8). 


\section{3. - Le Calcium}

Composition des divers organes et de la plante entiere.

Les différences de teneurs entre organes sont également très accentuées, surtout au cours du i er cycle, et les limbes sont beaucoup plus riches en Ca que les gaines et les tiges (tableau 4). A stade de croissance équivalent, la teneur en Ca augmente très nettement dans tous les organes au cours de l'année (figure 4) : ce fait, déjà observé lors des expériences précédentes (I957 et I958), semble donc indépendant des conditions climatiques. Ainsi, les plantes des derniers cycles sont environ dettx fois plus riches en $\mathrm{Ca}$ que celles du premier cycle.

\section{TABLEAU 8}

Teneurs en élíments minéraux des inflorescences de la fítuque En $g$ par $\mathrm{kg}$ de matière sèche

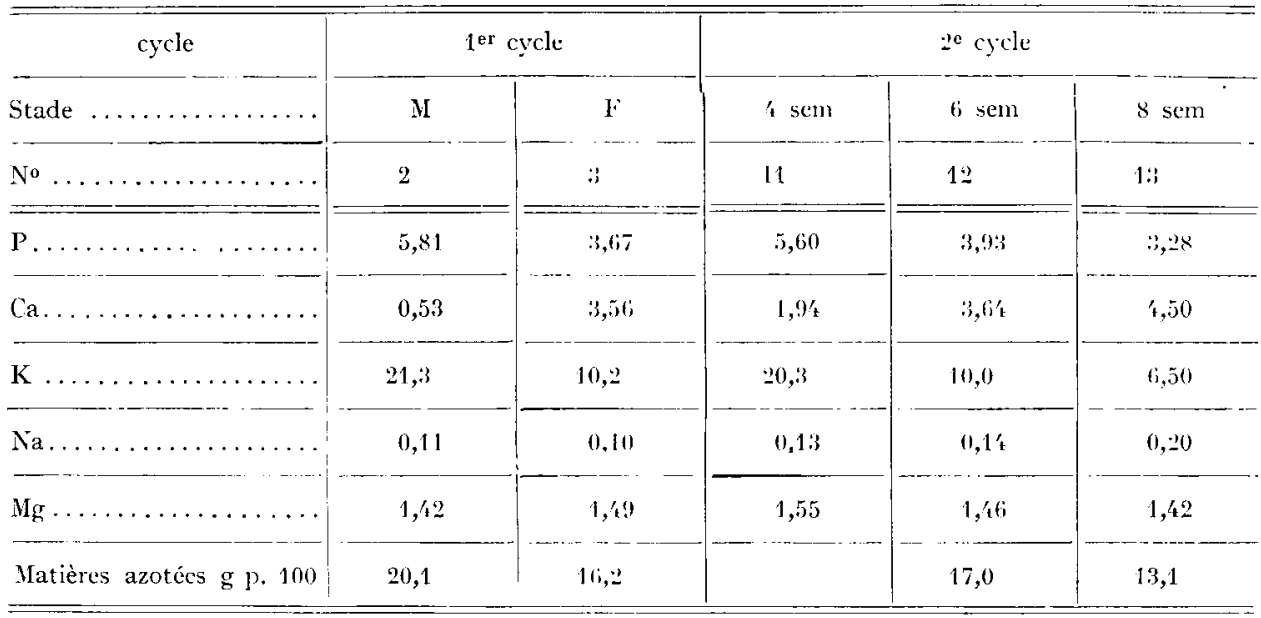

Infuence du stade de développement.

Au cours du $\mathrm{I}^{\mathrm{er}}$ cycle, comme nous l'avions déjà constaté en I957, les teneurs en $\mathrm{Ca}$ augmentent considérablement dans les limbes. Au cours des cycles suivants l'évolution est plus irrégulière, mais les limbes âgés sont en général plus riches en Ca que les limbes jeunes. Les gaines, au contraire, ont tendance à s'appauvrir en calcium en vieillissant tandis que les inflorescences s'enrichissent considérablement (tablea11 8). Par suite de ces variations opposées, l'influence du stade de développement est très irrégulière chez la plante entière : les teneurs en Ca passent par un maximum ou par un minimum au cours des différents cycles de végétation (figure 4).

\section{4. - Le Magnésium}

Nous retrouvons une règle générale déjà énoncée, à savoir que l'évolution du magnésium dans la plante est parallèle à celle du calcium. Les limbes sont toujours les organes les plus riches en Mg et, à stade de croissance équivalent, les teneurs en Mg 


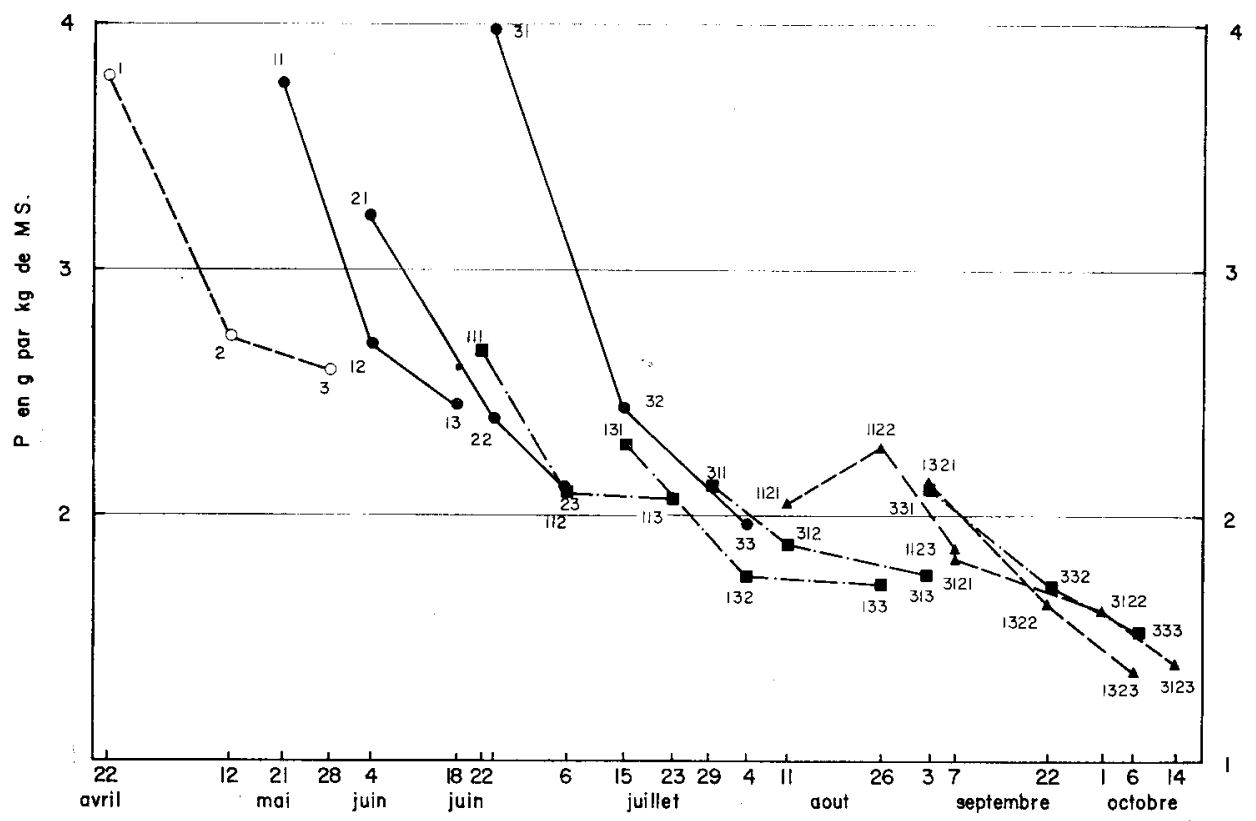

Fig. 3. -- Evolution de la teneur en phosphore de la fétuque des prés.

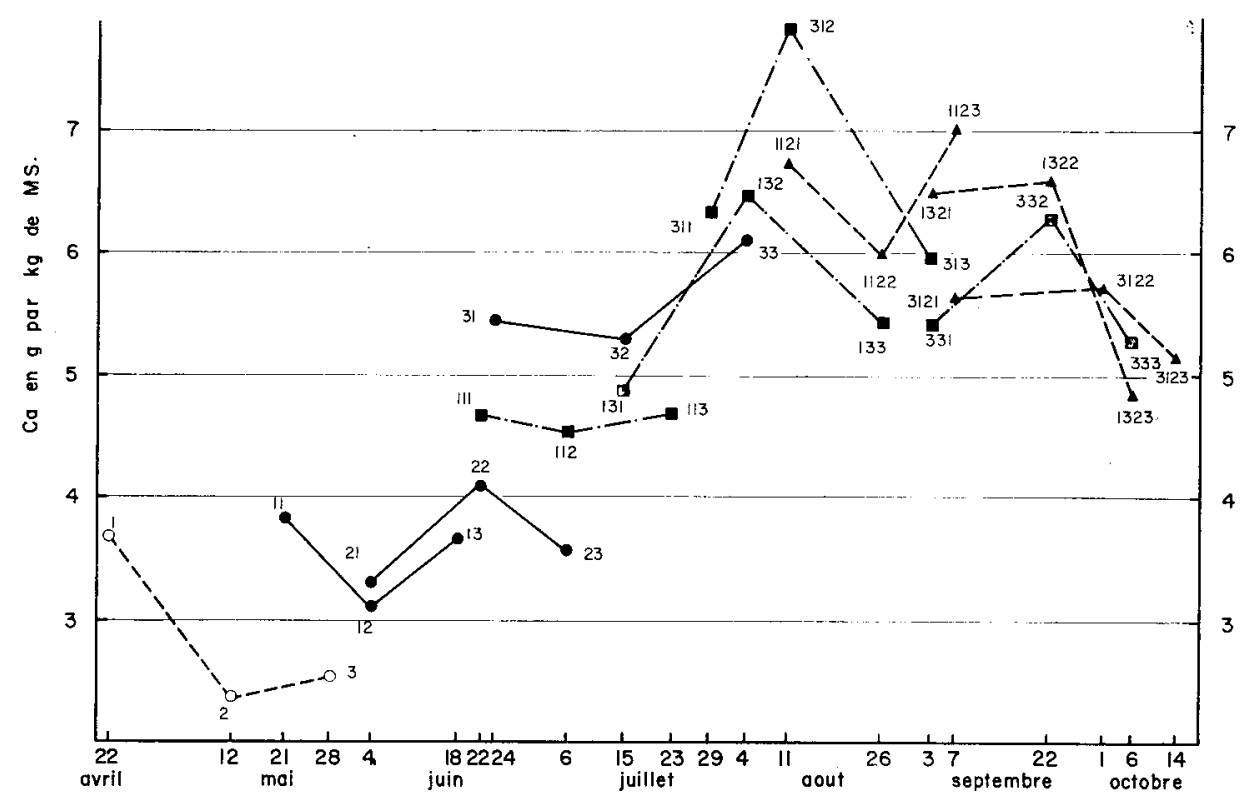

FIG. 4. - Evolution de la teneur en calcium de la fétuque des prés. 


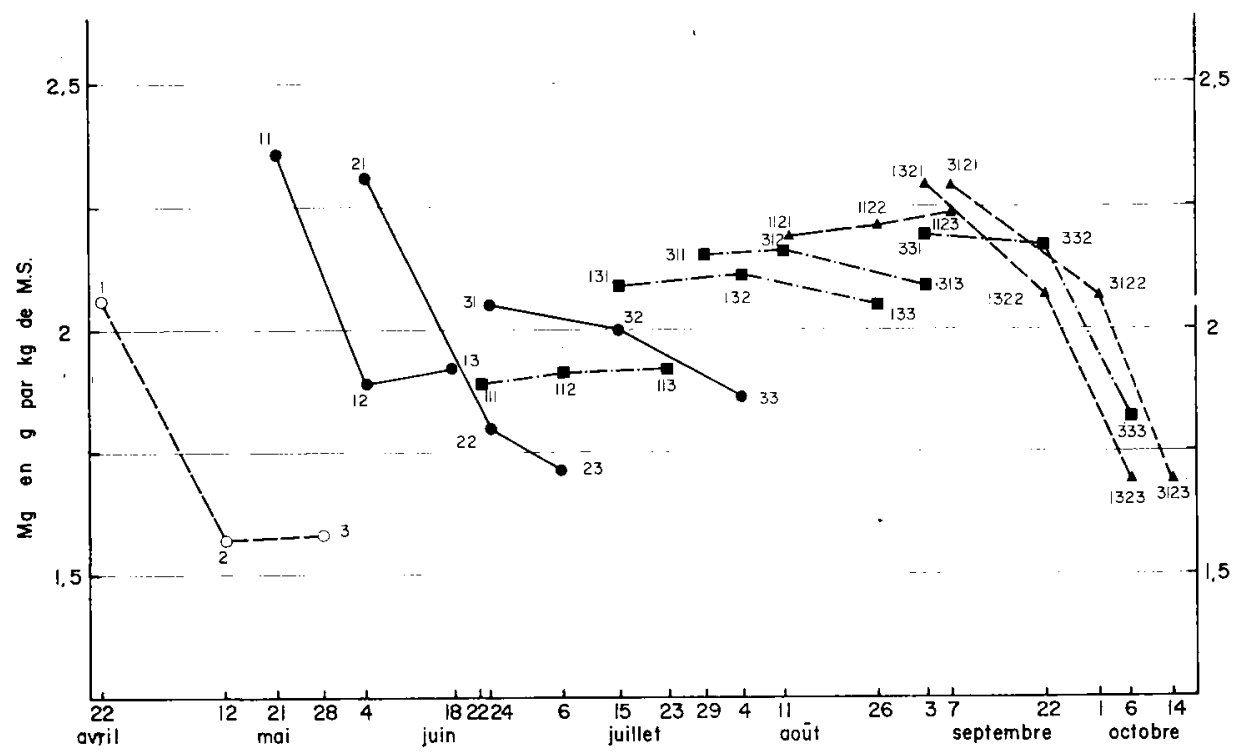

Fig. 5. - Evolution de la teneur en magnésizm de la fétuque des prés.

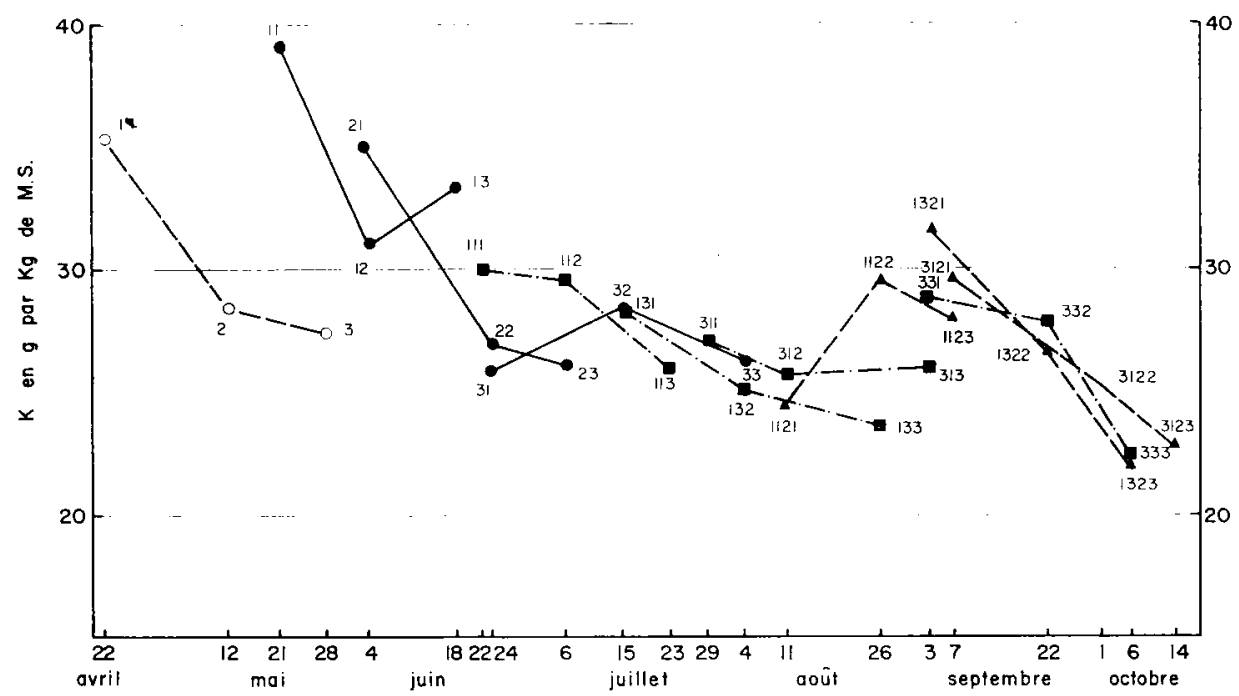

IIG. 6. - Evolution de la teneur en potassinm de la fétuque des prés. 
des limbes et de la plante entière augmentent sensiblement au cours de l'année. Les plantes âgées du I ${ }^{\text {er }}$ cycle sont les plus pauvres en $\mathrm{Mg}$.

L'influence du stade de développement est très accentuée durant les cycles de printemps et les derniers cycles d'automne, et se traduit par une diminution très nette de la teneur en $\mathrm{Mg}$ des plantes (tableau 7 et figure 7 ).

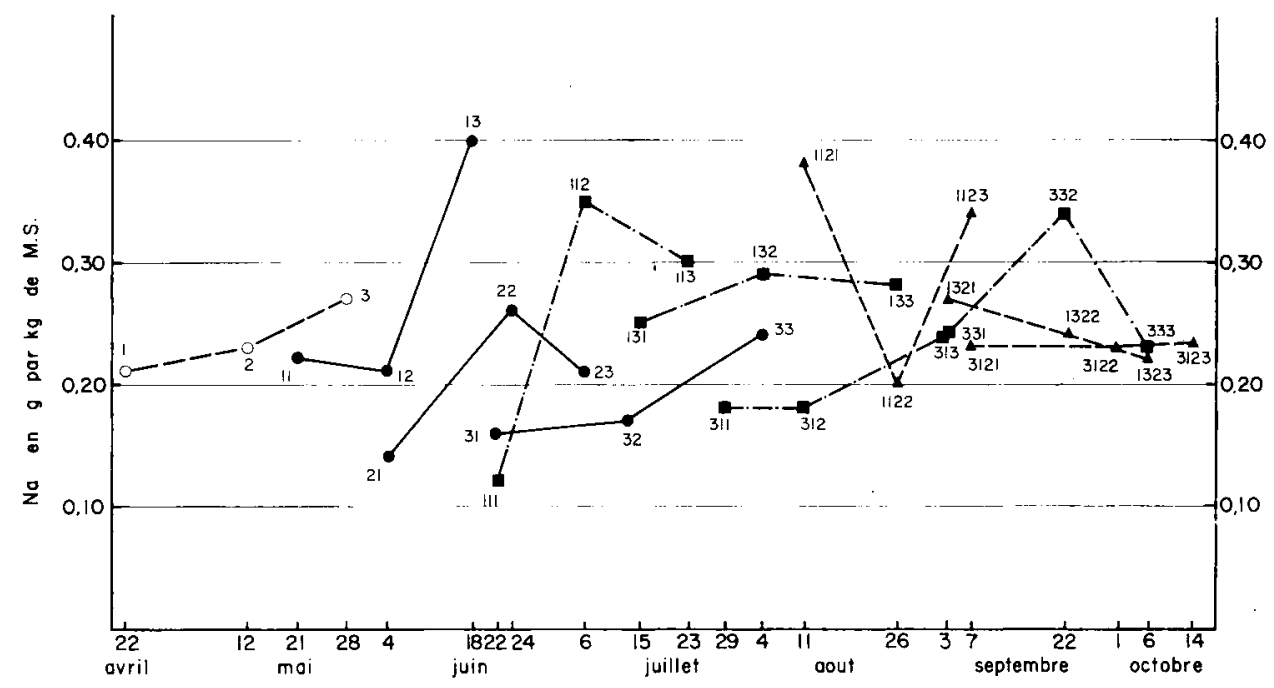

FIG. 7. - Evolution de la teneur en sodium de la fétuque des prés.

\section{5. - Le Potassium}

Les limbes sont toujours plus riches en $\mathrm{K}$ que les gaines et les tiges. A stade de développement équivalent, tous les organes s'appauvrissent en $\mathrm{K}$ au cours de l'année, et nous obtenons cette fois un minimum assez marqué à la mi-été, en accord avec STEW.ART et Hormes (I953). Les plantes jeunes des premiers cycles sont toujours les mieux pourvues en $\mathrm{K}$ (figure 5 ).

L'influence du stade de développement est très régulière durant tous les cycles de végétation : les teneurs en $\mathrm{K}$ de la plante entière et de ses divers organes diminuent au cours du vieillissement de la plante. Les inflorescences âgées sont particulièrement pauvres en $\mathrm{K}$ (tableaux 5 et 8 ).

\section{6. - Le Sodium}

Nous constatons de nouveau (GUEGUEN, 1959) que la fétuque est très pauvre en sodium. En général les gaines sont légèrement plus riches que les limbes en $\mathrm{Na}$, mais cette différence n'existe ici qu'au cours des premiers cycles de végétation (tableau 6).

Au cours de l'année, les variations des teneurs en Na de la plante entière et de ses divers organes sont très irrégulières et il n'existe pas de différence marquée entre le I $^{\text {er }}$ cycle et les cycles suivants. De même, bien que les teneurs en Na varient considérablement avec l'âge de la plante (les teneurs en $\mathrm{Na}$ peuvent doubler ou même tri- 
pler d'un stade de croissance au suivant), l'influence du stade de développement (figure 6) ne provoque pas de variations systématiquement orientées.

\section{DISCUSSION}

Connaissant les différences spécifiques fondamentales entre le Dactyle et la Fétuque, étudiées simultanément durant le premier cycle en I957, année normale du point de vue climatique (GUEGUEN, I959), nous pouvons essayer de définir l'influence propre du climat ou de la saison sur les teneurs en matières azotées et en éléments minéraux du Dactyle étudié en I958 (année très humide, en moyenne $73 \mathrm{~mm}$ de pluie par mois de mai à octobre) (GuEguen et Fauconneau, I960) et de la Fétuque étudiée en I959 (année très sèche).

En général, seule 1'influence conjuguée du climat et de la saison peut être observée. Toutefois, selon les constituants, nous pouvons constater une influence prépondérante: climatique sur les teneurs en matières azotées et en phosphore, saisonnière sur les teneurs en calcium, magnésium et potassium (avec également une action spécifique possible de la chaleur sur les concentrations en potassium).

Influence du climat. - La teneur en matières azotées des graminées est liée à leur croissance : quand celle-ci est ralentie par des conditions climatiques défavorables (sécheresse, froid, etc...) le vieillissement des organes s'accompagne d'une diminution importante des matières azotées. La différence entre le premier cycle etles cycles suivants est beaucoup moins accusée dans le cas de la fétuque i959 que dans le cas du dactyle I958 : la sécheresse a provoqué une diminution régulière et continue des teneurs en matières azotées, en particulier des limbes ; dans les plantes âgées de 6 semaines des $2^{\mathrm{e}}, 3^{\mathrm{e}}$ et $4^{\mathrm{e}}$ cycles, ceux-ci contiennent $\mathrm{I}_{4}$ - I5 $\mathrm{p}$. Ioo de matières azotées contre $18-24$ p. Ioo dans les limbes du dactyle.

Contrairement au dactyle, la fétuque s'est appauvrie régulièrement en $\mathrm{P}$ au cours de l'année et ceci est manifestement dû à l'influence prépondérante des facteurs climatiques, la forte sécheresse ayant diminué la disponibilité de $\mathrm{P}_{2} \mathrm{O}_{5}$ du sol. Ainsi, nous ne trouvons pas l'écart considérable enregistré entre le premier cycle et les suivants dans le cas du dactyle. Cette action de la sécheresse sur les teneurs en $\mathrm{P}$ des plantes a été vérifiée par de nombreux auteurs, en particulier par HomB (I953), ODELIEN (I948), WOODMAN et UNDERWOOD (I93I). Ces derniers auteurs observent des teneurs en $\mathrm{P}$ beaucoup plus faibles en I 929 , année sèche (en moyenne de $\mathrm{I}, 86$ à 3,60) qu'en I930, année humide (en moyenne de 4,02 à 5,I7).

Enfin, pour ce qui concerne le potassium, il semble que deux facteurs, la chaleur et la sécheresse, puissent agir en sens inverse. Les températures élevées causeraient une augmentation des teneurs en $\mathrm{K}$ des plantes (DIJKSHoorn et HaR'r ir957); nous constatons en effet que les teneurs moyennes en $\mathrm{K}$ des limbes de fétuque entre le début de l'épiaison et la floraison étaient respectivement de $33,3 \mathrm{~g}$ par $\mathrm{kg}$ en 1959 et de $26,7 \mathrm{~g}$ par $\mathrm{kg}$ en I957. D'autre part, au cours des cycles suivants, les teneurs moyennes en $\mathrm{K}$ des limbes étaient de 25 à $30 \mathrm{~g}$ par $\mathrm{kg}$ pour la fétuque en $\mathrm{I} 959$ contre $\mathrm{I}_{5}$ à $20 \mathrm{~g}$ par $\mathrm{kg}$ pour le dactyle en $\mathrm{I} 958$, bien que les teneurs des limbes de ces deux espèces étaient voisines en I957. Nous avions en outre constaté que les périodes particulièrement pluvieuses et chaudes de I 958 entrainaient un accroissement considérable des tèneurs en $\mathrm{K}$ du dactyle : ceci est probablement dû à l'action simultanée 
de la chaleur et de l'augmentation de la proportion de $\mathrm{K}$ assimilable du sol. En revanche, en I959, 1'action propre de la sécheresse, produisant une diminution de la disponibilité de $\mathrm{K}$ du sol, a été déterminante en fin de saison, oì les teneurs en $\mathrm{K}$ deviennent plus faibles dans la fétuque.

Infuence de la saison. - Indépendamment des conditions climatiques, les teneurs en $\mathrm{Ca}$ et $\mathrm{Mg}$ des plantes sont sujettes à des variations saisonnières très nettes et augmentent au cours de l'année. Cette influence de la saison a déjà été constatée par STEWART et Holmes (I953) pour Ca et Mg, et par Walshe et Conway ( I960) pour Mg. Ces derniers auteurs trouvent également que les plantes sont les plus pauvres en $\mathrm{Mg}$ au printemps et en fin d'automne.

Quant aux différences enregistrées entre les deux espèces pour ce qui concerne les variations en fonction du stade de développement, elles seraient dues à l'enrichissement en $\mathrm{Ca}$ des limbes beaucoup plus rapide et plus accentué chez la Fétuque que chez le Dactyle (GUEGUEN I959) ; c'est pourquoi l'appauvrissement régulier en fonction de l'âge de la plante ne s'observe pas dans le cas de la Fétuque.

Les teneuts en $\mathrm{K}$ des plantes semblent également sujettes à une influence saisonnière et diminuent régulièrement au cours de l'année quelles que soient les conditions climatiques.

Enfin, dans le cas du sodium, l'influence du climat et de la saison est toujours très irrégulière et les deux facteurs ne peuvent être dissociés. Il convient encore de souligner que le sodium est beaucoup plus abondant dans le Dactyle que dans la Fétuque (en moyenne 30 fois plus).

\section{Conséquences nutritionnelles et conclusions.}

Les expériences entreprises sur le Dactyle et la Fétuque durant ces trois années consécutives permettent de tirer un certain nombre de conclusions provisoires, d'une part sur les variations de la valeur nutritive de ces espèces fourragères, d'autre part sur le ou les modes d'exploitation à adopter.

Au cours du premier cycle, les variations des proportions des différents organes de la plante et l'évolution de leur composition chimique semblent peu sensibles aux facteurs externes. Aussi peut-on tirer des lois générales des variations de la valeur nutritive de la plante en fonction du stade de développement qui est le principal facteur de variation : la valeur nutritive en matières azotées et en éléments minéraux diminue rapidement au cours du premier cycle. C'est pourquoi il importe de récolter le premier cycle avant la montaison.

Au cours des cycles suivants, les dactyles et les fétuques sont constitués principalement de limbes dont la croissance dépend essentiellement des conditions climatiques: la composition chimique des plantes est très sensible aux variations des facteurs externes. Toutefois, sauf en ce qui concerne le phosphore en année de grande sécheresse, les plantes du premier cycle ont toujours une plus faible valeur nutritive en matières azotées et en éléments minéraux. Nous constatons que les deuxièmes cycles précoces (provenant $d u$ premier cycle interrompu avant la montaison) ont toujours des rendements élevés (entre 5 et 7 tonnes de matière sèche à l'hectare). En conséquence, compte tenu des différences de rendement en matière sèche observées entre cycles (fig. I), i1 importe de choisir un deuxième cycle de végétation pour récolter le maximum de fourrage de bonne valeur nutritive. 


\section{SUMMARY}

STUDY OF THE VARIATIONS OF CRUDE PROTEIN AND MINERAL CONTENTS OF MEADOW FESCLE.

The variations of the nitrogen and mineral ( $\mathrm{P}, \mathrm{Ca}, \mathrm{K}, \mathrm{Na}, \mathrm{Mg})$ contents of a forage grass, meadow fescue (Festuca pratensis), have been studied after the different parts of the plant were separated : leaf laminas, leaf sheaths, stems, inflorescences and debris. The fescue was cut at three different stages of development in the course of several vegetative cycles during the year I959.

The nitrogen was determined by the Kjeldahl method. The minerals were determined after calcination of the product : phosphorus by the colorimetric method with vanadate and calcium, sodium, potassium and magnesium by flame spectrophotometry.

This study completes work carried out in 1957 and $195^{8}$ on cocksfoot (Dactylis glomerata) and fescue, and makes it possible to confirm certain general rules already set forth.

In the course of the first cycle, the variations in the chemical composition of the plants are closely linked with the evolution of the leaf laminas/leaf sheaths + stems ratio and with the translocations caused by flowering. The nitrogen and mineral contents are higher in the leaf laminas than in the other organs and decrease with the age of the plant (apart from the Ca content which increases in the leaf laminas). The variations in the composition of plants in the first cycle seem to be not particularly sensitive to external factors (climate, soil...).

During the following cycles, fescue is composed chiefly of leaf laminas and the variations of the chemical composition of the plant are much more sensitive to climatic factors. The influence of the stage of development is more irregular than during the first cycle (See figures).

The comparison of results obtained on cocksfoot in 1958 (a very wet year) and on fescue in I959 (a very dry year) shows a preponderant climatic influence on the nitrogen and phosphorus contents of the plants (considerably decreased by drought) and a preponderant seasonal influence on the contents of calcium and magnesium (which increase during the year). The potassium content decreases regularly during the year in spite of the probable influence of high temperatures which would cause a passing increase of its concentration in the plants.

Plants in the first cycle have generally the lowest nutritive value in nitrogenous material and in mineral elements, and, taking into account the variations in yield observed, (fig. I) it is best to choose an early second cycle to bring in the maximum of forage of high nutritive value.

\section{RÉFÉRENCES BIBL,IOGRAPHIQUES}

DIJKSHOORN W., HART M. L., I957. Effect of alteration of temperature upon the cationic composition in perennial ryegrass. Neth. J. Agric. Sci., 5, $18-36$.

Fauconnenu G., Jarrigk R., I957. Composition chimique et valeur nutritive de l'herbe. Bull. Tech. Ing. Ser. Agric., 118.

Gueguen L., 1959. Etude des variations de la composition minérale de quelques espèces fourragères. Influence du stade de développement et du cycle de végétation. Ann. Zootech., 8, 245-268.

Gueguen L., Fauconneau G., ig6o. Etude sur les variations des teneurs en matières azotées et en éléments minéraux du dactyle. Ann. Zootech., 9, I57-1 79 .

Gueguen L., Rombauts P., 196r. Dosage du sodium, du potassium, du calcium et du magnésium par spectrophotométrie de flamme dans les aliments, le lait et les cxcreta. Ann. Biol. anin. Bioch. Biophys., 1, $80-97$

HomB 'T., 1952. Chemical composition and digestibility of grassland crops. Norges Landbr. Foringsforsok. Beretn., 71, 214 pp.

ODELIEN M., r942. The phosphorus content in hay on farms with heavier and lighter manuring. Tidsskr. Norske Landbr., 12, 1-8.

Soper K., Mitchell K. J., i956. The developmental anatony of perennial ryegrass (Lolium perenne L.). N. Z. J. Sci. Tech., 3\%, Sect. A, 484-504.

StEwart A. B., Holves W., 1953. Manuring of grassland. I. Some effects of heavy dressings of nitrogen on the mineral composition of grassland herbage. J. Sci. Food. Agric., 9, 40 I-4o8.

Walshe .I. J., Conway A., ig6o. Hypomagnesaemia in ruminants. Proc. of the eighth. Intern. Grassland Congress. (Reading) 548-55.3.

WoOdman H. E., UNDERWoOd E. J., I932. Nutritive value of pasture. 8. The influence of intensive fertilizing on the yield and composition of good permanent pasture (seasons I and 2). J.Agric. Sci., 22, 26-71. 